\title{
Walter Rudolf Hess
}

Aus seinem Leben und seiner Tätigkeit an der Medizinischen Fakultät der Universität Zürich

(100-Jahr-Feier seines Geburtstages: 14. März 1981)

Von Peter G. Waser

Es ist immer schwierig, über einen bedeutenden Mann zu berichten, auch wenn man einige Jahrzehnte mit ihm zusammen gelebt und gelegentlich auch gearbeitet hat. Wenn man wie ich zuerst sein Student war, später Assistent in einem nahen Forschungsinstitut und dann Kollege in der Fakultät, ist es besonders schwierig, kritisch zu bleiben mit aller Bewunderung für die außerordentliche wissenschaftliche Leistung, für die menschliche Größe und die kollegiale Verbundenheit, die er einem engen Kreis seiner Mitarbeiter und jungen Freunde entgegenbrachte. Was davon in der Erinnerung zurückbleibt, ist weniger das Sachliche, die genauen Daten des Lebens, sondern der ganz persönliche menschliche Aspekt einer Begegnung, die Spuren hinterläßt, Eindrücke macht und Anregungen vermittelt. So ist mein Bericht sicher lückenhaft, einseitig und persönlich - aber indem wir eines uns prägenden Kollegen und Mitmenschen gedenken, geschieht es aus einer subjektiven, unvollständigen Sicht, die zum Gesamtbild eines Forschers und Menschen gehört.

Zuerst kurz einige biographische Daten: W. R. Hess wurde am 17. März 1881 in Frauenfeld (Kt.Thurgau) als Sohn eines Mittelschullehrers für Physik geboren. Er verlebte dort eine glückliche Jugend in Naturverbundenheit, kam bereits durch seinen Vater in Kontakt mit naturwissenschaftlichen Problemen, besonders in der Physik und Meteorologie, und mit einigen technischen Betrieben der erwachenden Industrie. Nach dem Gymnasium begann er sein Medizinstudium in Lausanne in einem kulturell und sprachlich französisch orientierten Landesteil der Schweiz. Doch waren auch andere Medizinschulen wie Bern, Zürich und vor allem Berlin und Kiel wichtige Ausbildungsstätten, die nicht nur fachlich, sondern vor allem kulturell auf den jungen Medizinstudenten großen Eindruck machten. Neben Konzert- und Theatervorstellungen besuchte er auch die Museen. Vor allem die Meeresaquarien und ein astronomisches Institut mit einem 
Riesenfernrohr hinterließen einen tiefen Eindruck. Hess schreibt selbst: «Es beschäftigt mich die Frage der Unendlichkeit, das, was sich hinter den sichtbaren Sternen befindet!» Also beschäftigte ihn schon von Anfang an die Frage, was hinter den Objekten steht, nicht nur das, was in der Natur einfach beobachtet wird, sondern die Zusammenhänge dahinter oder darüber hinaus, die Kraft und die Organisation, welche die äußere Welt zusammenhalten und bewegen. Dazu ein Beispiel: Im Präpariersaal des Zürcher Anatomischen Institutes wurde eine arterielle Anomalie demonstriert, die Hess als Student dazu bewegte sich zu fragen, wie die Natur eine Optimierung der Gewebedurchblutung erreiche. Wenn sich eine Arterie in zwei Äste aufspaltet, muß bei konstantem Gesamtvolumen der Hauptarterie und ihrer beiden Äste der Gesamtwiderstand ein Minimum werden. Das einfache mathematische Problem wurde zusammen mit dem Zürcher Mathematiker Prof. Rudolf Fueter gelöst und experimentell bestätigt. Wir mußten später als Studenten der Naturwissenschaften dieses Problem in der Vorlesung über höhere Mathematik bearbeiten, und es ist mir deshalb durch seine Klarheit und Logik in Erinnerung geblieben.

Der ehemalige geniale Gerichtsmediziner unserer Fakultät Prof. Heinrich Zangger beurteilte die kurze Abhandlung: «Eine mechanisch bedingte Gesetzmäßigkeit im Bau des Blutgefäßsystems» sehr positiv - und förderte wohl damit die wissenschaftlichen Interessen des jungen Studenten. Nach einigen klinischen Auslandsemestern, wie sie damals noch üblich - und möglich! - waren, legte Hess ein Staatsexamen für Medizin in Zürich 1905 ohne Schwierigkeiten ab.

Leider konnte Hess aus finanziellen Gründen nicht sofort als Assistent am Physiologischen Institut tätig sein, wie er sich das wünschte. Damals reichte eine Assistentenbesoldung für Wissenschafter auch bei einfachster Lebensführung nicht aus, und eigene Mittel standen nach dem Studium nicht zur Verfügung. So begann er seine Ausbildung im Allgemeinen Krankenhaus in Münsterlingen am Bodensee. Doch war der chirurgisch orientierte Chef (Konrad Brunner) sehr streng, die Freizeit knapp. Sie reichte daher nur zur Ausarbeitung einer selbstgewählten Dissertation über: «Viscosität des Blutes und Herzarbeit» (1906). Das damals entwickelte Viscosimeter (eine relative Bestimmungsmethode zum Vergleich der Viskositäten von Blut und Wasser) benutzten wir alle als Medizinstudenten im Physiologie-Kurs während Jahrzehnten! Die eigentliche Ausbildung erfolgte nun in der Zürcher Augenklinik (Prof. Otto Haab) zum Facharzt für Ophthalmologie, wobei sich Hess wieder für ein physiologisches Problem, 


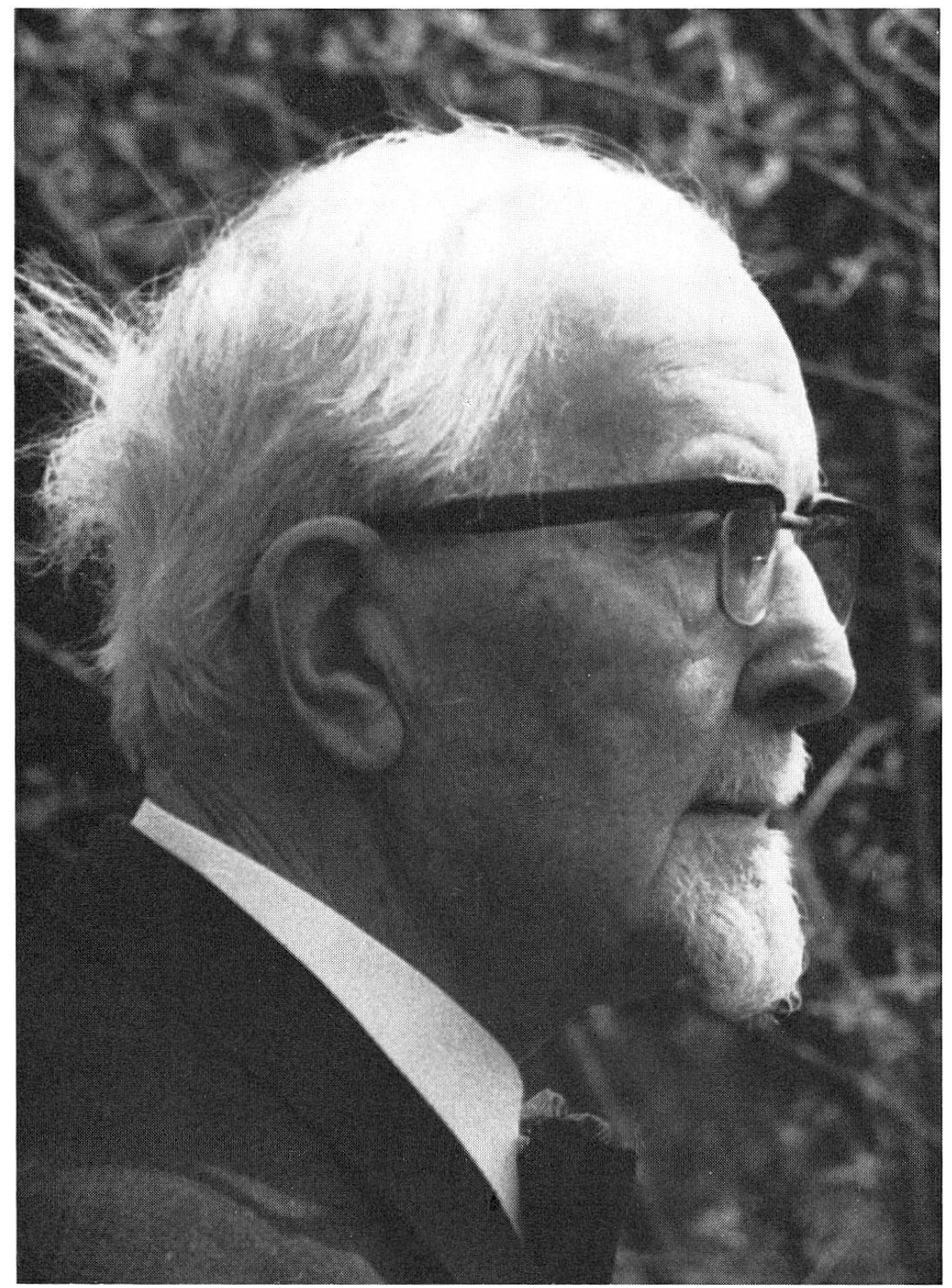

Walter Rudolf Hess

1881-1973

die Koordination der Bewegung der Augen und die Diagnostik der Augenmuskellähmungen, interessierte und ein entsprechendes Gerät zur genauen Messung dieser Funktion nach einem neuen Prinzip konstruierte.

Nach einer weiteren praktischen Ausbildung in Paris in Neurologie, Venerologie und innerer Medizin eröffnete Hess 1908 eine Praxis für 
Augenheilkunde in Rapperswil. Das gute Einkommen ermöglichte ihm endlich die Heirat. Aber alle praktischen Tätigkeiten, auch die nebenamtliche Betreuung einer klinischen Abteilung für Augenkranke im Kantonsspital Glarus, befriedigten ihn nicht, so daß er im Herbst 1912 nach Zürich zurückkehrte und Assistent beim Physiologen Prof. Justus Gaule, einem Schüler Carl Ludwigs, wurde. Der sich zunehmend verschlimmernde psychische Zustand seines Chefs brachte rasch eine steigende Belastung im Unterricht und im Kurs. Dazu reichte das Assistentengehalt für die junge Familie, inzwischen mit einer Tochter und einem Sohn, nur für eine äußerst sparsame Lebensführung. Eine Erfindung zur stereoskopischen Betrachtung photographischer Bilder in räumlicher Dimension sollte finanzielle Hilfe bringen.

Doch dann kam 1914 der Weltkrieg und verlagerte die medizinische Tätigkeit des Sanitäts-Hauptmannes Hess in die Armee, ohne die Möglichkeit, neben den gelegentlichen Urlauben für dèn Medizinerunterricht noch Forschung zu betreiben. Ein ganzjähriger Auslandsurlaub (1915/16) in Bonn beim Physiologen Prof. Max Verworn erlaubte trotzdem eine wesentliche Weiterbildung und Forschungsarbeit im Kreislaufgebiet über die Regulierung des Blutdruckes und Probleme der Haemodynamik, die später in Zürich fortgesetzt wurde. Die Habilitation (1913) hatte schon als Thema die physikalisch-biologische Basis der Zusammenhänge verschiedener Faktoren für die Kreislaufregulierung enthalten! Der damalige Internist war anscheinend bei der Beurteilung der Habilitationsarbeit über die mathematischen Formulierungen «als der Physiologie ferne», wie er sagte, unglücklich, wahrscheinlich weil die mathematische Interpretation biologischer Vorgänge für den praktischen Mediziner neu war.

Die Entscheidung über die Zukunft von Hess fiel im Wintersemester 1916/17 durch den unerwarteten Rücktritt von Prof. Gaule. Zuerst las Hess die ganze Physiologie-Vorlesung vertretungsweise, als Lückenbüßer, wie er selbst sagte, lehnte aber ein Extraordinariat unter einem neuen Chef, der aus Deutschland berufen worden wäre, ab. Im September 1917 wurde er dann durch die Fakultät zögernd, aber durch den Erziehungs- und Regierungsrat einstimmig, zum Nachfolger und Ordinarius gewählt. Bestimmend war damals wohl der Erziehungsdirektor, Regierungsrat Mousson, der nicht nur die Studenten über die Vorlesung befragte, sondern einmal selbst die sehr lebendige Vorlesung über Experimentalphysiologie des Privatdozenten Hess besuchte und davon sehr beeindruckt war. Die Antrittsrede vom 15. Juni 1918 befaßte sich mit der «Zweckmäßigkeit im Blutkreislauf». 
Die Lehrtätigkeit möchte ich aus eigener Erfahrung beurteilen. Meine Generation begann das Medizinstudium vor dem Zweiten Weltkrieg und kam während der Aktivdienstjahre in die klinische Ausbildung. Wir waren gerade während dieser Übergangszeit drei Semester lang mit Physiologie und Anatomie beschäftigt. Die Vorlesung war für uns anregend, lehrreich, systematisch. Sie wurde begleitet von zahlreichen Experimenten und Modellen, die uns das funktionelle Äquivalent der detaillierten Vorlesung über die Anatomie des menschlichen Körpers gaben. In dieser Hinsicht waren die Stunden im alten Hörsaal des Physiologischen Institutes für mich ein weiter Ausblick in die eigentlichen Lebensvorgänge, die ich später in der Klinik in einer ganz anderen Perspektive, mit Störungen oder Abnormitäten, kennenlernen sollte. Neu, und für viele auch ein vergnügliches Schauspiel, waren die Filme ohne Ende, bei denen der gleiche funktionelle Ablauf einer Magen-Darm-Bewegung oder der Herz-Kreislauf-Tätigkeit sich immer wiederholte. Da das Endstück des 9-mm-Schmalfilms an den Anfang der Sequenz angeklebt war - eine Schlaufe ohne Ende -, wiederholte sich der gleiche Vorgang stereotyp, natürlich von Hess mit lebhaftem Kommentar begleitet, bis wir die Sache ganz kapiert hatten.

Hess war ein didaktisch begabter Lehrer, der es verstand, uns auch schwierige Dinge in anschaulicher Weise verständlich zu machen und uns zu eigenem selbständigen Denken und Experimentieren anleitete. Wenn dann aber die Filme der Katzenversuche gezeigt wurden, bei denen eine elektrische Hirnreizung immer wieder die gleiche Wirkung, z. B. Erbrechen oder Defäkation oder Schlaf, dann wieder Erregung hervorrief, wurde es still im Auditorium. Denn mancher überlegte sich, was nun wirklich Entscheidung und freier Wille sei, wenn ein Druck auf eine Taste über eine Hirnreizung ein Tier zu einem bestimmten, immer wieder gleichartigen Verhalten bringt, ohne daß das Tier diese Tätigkeit eigentlich ausführen will. Diese Experimente waren überzeugend, und wir jungen Medizinstudenten ahnten, welche großartigen Probleme unser Professor in Bearbeitung hatte, um die funktionellen Zentren des Gehirns zu verstehen.

Auch der Experimentierkurs war für uns eine wertvolle Instruktion. Er war gut organisiert, von Assistenten überwacht und hatte zahlreiche interessante Arbeitsplätze aus den verschiedenen Gebieten der Physiologie. Es wurden dabei nicht immer leichte Aufgaben gestellt. «Papa Hess», wie wir ihn unter uns nannten, mit Baskenmütze auf dem weißen Haupt von Platz zu Platz schreitend, befragte uns, demonstrierte, diskutierte oft über Themen, die weit entfernt vom Fachgebiet lagen, aber von großem menschli- 
chem Interesse waren: über Ethik, Leben, Krankheit und Tod, oder über die Notwendigkeit von Tierversuchen in der Medizin und in den biologischen Wissenschaften und über viel anderes mehr. Das Thema über Tierversuche wurde oft angesprochen, weil Hess fortwährend von den Vivisektionsgegnern verfolgt wurde und Mühe hatte, ihre gehässigen Angriffe abzuwehren. Diese Wirrköpfe hätten seine Forschungsarbeit verunmöglicht, wenn sie dazu den richtigen demagogischen Einfluß auf die Politiker gefunden hätten.

Nicht zu vergessen ist, daß Hess schon 1922-24 Dekan der Medizinischen Fakultät der Universität Zürich war und später auch wesentlichen Anteil an deren Entwicklung zur heutigen Leistungsfähigkeit auf hohem Niveau hatte. Während der ersten 15 Jahre war in seiner Lehrverpflichtung auch die Physiologische Chemie eingechlossen. Erst 1932 wurde ein separater Lehrstuhl für Pysiologische Chemie errichtet. Zuerst war er mit einer ungeeigneten Persönlichkeit besetzt, aber später wurde er unter Prof. Franz Leuthart als Biochemie zum neuen Grundpfeiler der Medizin und Biologie ausgebaut.

Die Gründung des hochalpinen Forschungsinstitutes Jungfraujoch wurde durch Hess als Präsident einer Kommission der Schweizerischen Naturforschenden Gesellschaft gefördert. Auch war Hess Mitbegründer der Schweizerischen Physiologischen Gesellschaft und deren wissenschaftlichen Zeitschrift. Er organisierte zudem 1938 als Tagungspräsident den Internationalen Kongreß für Physiologie, Physiologische Chemie und Pharmakologie in Zürich. Hess pflegte einen regen Kontakt mit vielen Wissenschaftern. Er war auch oft in Zürich mit Paul Karrer und Leopold Ruscizka zusammen.

Die Forschung hat Hess während seiner ganzen akademischen Tätigkeit voll in Anspruch genommen. Neben der anstrengenden Lehrtätigkeit war sie nur möglich durch gute Planung in einem speziellen, begrenzten Wissensgebiet und mit dem vollen Einsatz aller Kräfte. Bei dem begrenzten Personalund Finanzetat der damaligen Zeit konnte dies nur durch Bildung eines über lange Zeit zusammenarbeitenden Teams, aus meistens nicht studierten Technikern, ermöglicht werden, die über Jahrzehnte beisammen blieben und die neue Forschungstechnik weiter ausbauten (Frl. Verena Bucher, Herr Max Jenny, Frl. Anna Jaussi und Frau Mina Eugster). Dazu kamen während längerer Zeitperioden jüngere Forscher wie Ernst Rothlin und Alfred Fleisch, später dann Oskar Wyss, Marcel Monnier, Werner Koella, Konrad Akert und Robert Hunsperger als Oberassistenten. Alle wurden Professoren für Physiologie in Zürich, Basel oder an anderen Universitäten, zwei von ihnen Nachfolger auf dem Lehrstuhl und Leiter des Physiologi- 
schen Institutes in Zürich. Eine große Zahl von Assistenten und Doktoranden nahm nur während kürzerer Zeit an einem wissenschaftlichen Problem teil. Ihre Beiträge waren wichtige Steine im großen Gebäude des Forschungsplanes, der während Jahrzehnten konsequent und intensiv verfolgt wurde. So war auch eine maximale Effizienz der eingesetzten Arbeitskräfte und der damals, vor der Gründung des Schweizerischen Nationalfonds, noch beschränkten staatlichen Forschungsmittel möglich. Neben kleineren Stiftungen und einer Unterstützung durch die Industrie, war es vor allem die Rockefeller Foundation, die wiederholt finanziell mithalf. Zahlreiche Forschungsurlaube ermöglichten es Hess, seine Resultate in Monographien zusammenzufassen.

Das Forschungsinteresse war vor 1930 auf den Blutdruck, die Haemodynamik und vor allem auf die Regulation von Kreislauf und Atmung konzentriert. Es wechselte dann, entsprechend der Verfolgung der zentralen Steuermechanismen, und konzentrierte sich nach Beschreibung der funktionellen Organisation des vegetativen Nervensystems auf das Zwischenhirn. Dieses zentrale, subcorticale Gebiet zur Regulation der Tätigkeit der inneren Organe wurde mit der hauseigenen Technik während über 20 Jahren unermüdlich erforscht. Durch Reizung und Ausschaltung diskreter, kleiner Hirngebiete, ihre genaue histologische Lokalisation und Eintragung auf Karten der Hirnquerschnitte wurde der Zusammenhang mit den beobachteten peripheren Wirkungen am Tier festgestellt und damit die funktionelle Organisation dieses Hirnteiles, des Diencephalon, aufgeklärt. Diese mühsame Analyse eines dichten, neuronalen Hirngebietes und die Methode waren so erfolgreich, daß später das ganze Gehirn von anderen Forschungsgruppen bei verschiedenen Tierspezies untersucht und in verschiedenen Ebenen kartographiert wurde mit allen Angaben der Reizpunkte und der eintretenden Wirkungen. Auf dieser Grundlage beschäftigen sich heute die Neurochemiker mit den vielfältigen Formen chemischer Impulsübertragung durch Neurotransmittoren und Neurohormone, die Neurophysiologen mit den komplizierten neuronalen Verbindungen, dem Schaltplan der Neurone, und die Molekularbiologen mit den Vorgängen, welche das Lernen und die Gedächtnisbildung ermöglichen. Das Verständnis für das Wesen der Denkvorgänge auf molekularer Ebene ist dadurch in Sichtweite gekommen, zum Teil auch auf der Grundlage der Hessschen Forschungsresultate. Hess hat für seine Leistungen auch zahlreiche Ehrungen erhalten: Ehrendoktorate, Mitgliedschaft in Akademien, nationale und internationale Preise und insbesondere 1949 den Nobelpreis für Physiologie und Medizin. 
Nach der Emeritierung (1951) bearbeitete Hess weiter sein immenses Forschungsmaterial und publizierte die Zusammenfassung der Resultate in verschiedenen Monographien. Sein Interesse wendete sich nun noch höheren Leistungen des Gehirns zu: der Psyche! So verfaßte er ein bemerkenswertes Buch über «Psychologie in biologischer Sicht» (1962), das bald in verschiedene Weltsprachen übersetzt wurde. Wir durften als junge Forscher bei der zweiten, erweiterten Auflage (1968) in einem interdisziplinären Gespräch über ausgewählte Themen mitarbeiten. Es war für uns natürlich äußerst interessant, anregend, ehrenhaft, aber auch amüsant, mit unserem verehrten und geliebten Lehrer nochmals diesen letzten Gedankenaustausch zu haben. So wurden viele von uns ausgebildet und geprägt durch die großartige Forschung, aber auch erzogen und gefördert durch den persönlichen Kontakt mit diesem selbstkritischen, exakt denkenden und logisch handelnden Mann. Den tiefsten Eindruck machen uns heute wohl kaum die zahlreichen Ehrungen, die ihm zuteil wurden, sondern die Tatsache, daß Hess als «Selfmade man» sich ohne Lehrer oder Schule zum Forscher entwickelte und seinen Weg zum Erfolg ohne äußere Hilfe mit einer unglaublichen Energie und Selbstdisziplin allein ging. Dadurch bleibt er für uns ein großes Vorbild.

Ein vollständiges Verzeichnis der wissenschaftlichen Arbeiten von W. R. Hess ist im Buch «Biological Order and Brain Organization, Selected Works of W.R.Hess», edited by K. Akert, Springer Verlag, 1981, enthalten.

\section{Summary}

The life and scientific career of Walter Rudolf Hess is described from a personal viewpoint of a former student and later colleague of the medical faculty of the University of Zürich. His selfeducation, his unlimited energy and discipline in hard research work led him to pioneering new techniques and knowledge of brain functions and behaviour. He was always held in the highest esteem throughout his life by his faithful and successful pupils in different fields of biological and medical research and officially honoured by the endowment of the Nobelprize in Physiology and Medicine in 1949.

Prof. Dr. med. et phil. II Peter G. Waser

Pharmakologisches Institut der Universität Zürich

Gloriastraße 32

CH-8006 Zürich 